\title{
Optimal Asset Allocation: Risk and Information Uncertainty
}

\author{
Sheung Chi Phillip Yam ${ }^{\mathrm{a}}$, Hailiang Yang ${ }^{\mathrm{b}}$, Fei Lung Yuen ${ }^{\mathrm{c}, *}$ \\ ${ }^{a}$ Department of Statistics, \\ The Chinese University of Hong Kong. Shatin, Hong Kong. \\ ${ }^{b}$ Department of Statistics and Actuarial Science, \\ The University of Hong Kong. Pokfulam Road, Hong Kong. \\ ${ }^{c}$ Department of Mathematics and Statistics, \\ Hang Seng Management College. Hang Shin Link, Shatin, Hong Kong
}

\begin{abstract}
In asset allocation problem, the distribution of the assets is usually assumed to be known in order to identify the optimal portfolio. In practice, we need to estimate their distribution. The estimations are not necessarily accurate and it is known as the uncertainty problem. Many researches show that most people are uncertainty aversion and this affects their investment strategy. In this article, we consider risk and information uncertainty under a common asset allocation framework. The effects of risk premium and covariance uncertainty are demonstrated by the worst scenario in a set of measures generated by a relative entropy constraint. The nature of the uncertainty and its impacts on the asset allocation are discussed.
\end{abstract}

Keywords: Uncertainty modelling, Uncertainty measure, Asset allocation, Mean-variance approach, Relative entropy.

\section{Introduction}

Mean-variance approach introduced by Markowitz (1952) inspires numerous studies in the asset allocation problem. The key idea of mean-variance approach is to consider the optimal portfolio selection as a balance between

\footnotetext{
*Corresponding Author, Telephone: +852 3963 5243, Fax: +852 39635339

Email addresses: scpyam@sta.cuhk.edu.hk (Sheung Chi Phillip Yam), hlyang@hku.hk (Hailiang Yang), kevinyuen@hsmc.edu.hk (Fei Lung Yuen)
} 
reward and risk, where they are quantified using expected value and variance (standard deviation) of the portfolio return. This seminal idea attracts a lot of attention and it becomes the foundation of many researches in this area. It plays an important role in the development of some important concepts in finance, such as efficient frontier (Merton, 1972) and the capital asset pricing model (Sharpe, 1964). Researchers investigate and modify the basic meanvariance model and try to obtain more practical results on asset allocation. For example, Li et al. (1998) minimizes the probability of a significant loss and studies the asset allocation problem in a multi-period model. The multiperiod framework is also applied on a mean-variance formulation in $\mathrm{Li}$ and Ng (2000). Bertsimas et al. (2004) uses a conditional expected loss to replace variance for portfolio optimization. In these researches, the distribution of the assets are assumed to be known by the investors with a full certainty.

Involving uncertainty is another direction of study in asset allocation problem. In the real market, the exact distributions of the risky assets are normally unknown. We can only estimate the distribution of the assets by the historical data and personal experience. The estimation is not necessarily accurate and it is known as the uncertainty (or ambiguity) of the assets' distribution. The famous Ellsberg paradox suggests that people are uncertainty averse. There are many researches about risk. It is interesting and important to know more about the nature of uncertainty and understand its effects on decision making. Various models are suggested to analyse the effect of uncertainty. Different researchers have different focuses on their uncertainty model. Quiggin (1982), Schmeidler (1989), Tversky and Kahneman (1992) use a non-additive probability setting to model the uncertainty aversion character. One key feature of these models is that the result is consistent with stochastic dominance and so they have great contributions to the development of behavioral finance. Some researches pay more attention to the mathematical and statistical nature of parameter uncertainty. Yaari (1987) constructs a dual theory to demonstrate uncertainty aversion of agents. DeMiguel and Nogales (2009) replaces mean and variance with more robust reward and risk measures to increase the consistency of the mathematical results. Bodnar et al. (2013) considers statistical errors on the parameters. Stochastic models can also be introduced for the uncertain parameters. Gennotte (1986) identifies the optimal investment strategy with a stochastic model on covariance matrix. Klibanoff et al. (2005) uses the idea of utility function to demonstrate uncertainty aversion. Huang and Ying (2013) applies the concept of fuzzy logic to model asset return. 
Another way to model uncertainty is to consider a set of parameters (which represents different scenarios or distributions) rather than a point estimation of the parameters. It is sometimes known as the robust optimization. Bertsimas et al. (2007) provides more details and mathematical setting of this approach. As the actual parameters are unknown, by using a set of parameters, a more robust result can be obtained. This idea is commonly used in engineering, operations research, financial economics and many other subjects. There are different ways to generate a set of parameters. Tütüncü and Koenig (2004), Epstein and Schneider (2008) consider intervals which are likely to include the actual parameters. Gregory et al. (2011) further introduces a boundary for the number of uncertainty parameters. Hansen and Sargent (2001), Lim and Shanthikumar (2007) use the concept of relative entropy to generate the set of parameters. Schtellà and Recchia (2013) proposes more methods for generating a range of parameters.

There are various statistical methods to estimate the value of unknown parameters. The true value of the parameters is expected to be around the estimator. When a set of probability measures is used to model uncertainty, it should be consistent with the statistical results and not too far away from the estimation. Relative entropy measures the difference between distributions (or probability measures). Hence, it can reasonably be used to generate an uncertainty set of parameters. It is done by setting a constraint on the deviation between the best estimated measure and uncertainty measures. The worst scenario in the uncertainty set is then chosen to illustrate the uncertainty aversion of the investors. Relative entropy has been used in various optimization problems, including asset allocation. Hansen and Sargent (2001) uses relative entropy to model uncertainty and obtains the deterministic optimal investment strategy. Calafiore (2007) considers a discrete number of scenarios and studies the computational algorithm of solving the problem. Yuen and Yang (2012) replaces variance by expected loss to measure investment risk and identify the relationship between risk and uncertainty. Existing researches mainly focus on the uncertainty of risk premia and its impacts on the investment strategy. Here, we introduce a model on the uncertainty of the interaction between the returns of different assets in order to study its mathematical and financial properties in asset allocation problem.

In this article, we study the asset allocation problem with uncertainty using the mean-variance approach. We apply the multivariate normal distribution to model the returns of the assets. People are assumed to be uncertainty 
averse. They are conservative when they make a decision in an uncertain situation. Relative entropy is used to generate a set of probability measures which demonstrates the uncertainty of the parameters. The set is found to have some nice mathematical properties which are important in modelling covariance uncertainty. Different measures in the set refer to different scenarios in the market. The worst scenario is used to study the uncertainty aversion characteristic of the agents. Through this model construction, we can obtain the properties of the worst scenario and the corresponding optimal investment strategy. In the following, we focus on expected return uncertainty in Section 2 and covariance uncertainty in Section 3. In Section 4, we present more mathematical details of the model and consider the two sources of uncertainty together. In Section 5, numerical examples are used to illustrate the ideas of our model. More characteristics of the model are also discussed.

\section{Model Formulation and Uncertainty on Risk Premia}

We assume that there are $n$ risky assets in the market. Their returns follow multivariate normal distribution. Let $r_{f}$ be the risk-free rate, $\mu$ and $V$ be the risk premium and the covariance matrix of the risky assets' returns under the physical probability measure $P$, respectively. Here, $P$ represents the best estimated market environment. Hence, $\mu$ and $V$ are the best estimations towards the parameters of returns of these assets by the investors using all available information, including historical data, news, their knowledge and etc. Under mean-variance approach, they are linked to the reward and the risk of investment. We also assume that there are no redundant risky assets in the market. Hence, $V$ is symmetric and positive definite. For square matrices $M$ and $N$ with the same dimension, we write $M \succ N(M \succeq N)$, if, $M-N$ is positive definite (semi-definite). We have $V \succ 0$.

It is possible for the estimated parameters, $\mu$ and $V$, to have large differences with the actual parameters (uncertainty). That means, the expected investment performance and the inter-relationship of these assets are different from our estimation. The optimal portfolio induced by these two parameters can be inappropriate. Apart from considering the risk of the assets' return, we also need to study the effects of uncertainty of the model parameters. The investors might consider a range of scenarios based on their estimations to identify the potential loss if the market deviates from their prediction. In our model, $\mathcal{Q}$ denotes the set of uncertainty measures representing these sce- 
narios. It illustrates the effects of uncertainty in the decision making process. Let $p(x)$ and $q(x)$ be the probability density functions of the risky assets' returns under measures $P$ and $Q \in \mathcal{Q}$, respectively. If there is no uncertainty on the parameters, measure $P$ is used directly to study the problem.

We can now construct the set $\mathcal{Q}$ using the idea that the measures in $\mathcal{Q}$ should not greatly deviate from $P$. Relative entropy is used to measure the deviation between two probability measures. We assume that the relative entropy of all measures in the uncertainty set $\mathcal{Q}$ with respect to $P$ is not greater than a positive constant $K$. That is,

$$
K L(Q, P):=\int q(x) \ln \frac{q(x)}{p(x)} d x \leq K, \quad \forall Q \in \mathcal{Q} .
$$

The parameter $K$ depends on the investors' confidence on the available information and their opinions on the market when they make an investment decision. It is greater when they are more conservative in investing these risky assets. The uncertainty set needs not be large enough to cover all possible (extreme) scenarios that the investors can imagine. However, it can cover the adverse scenarios that the investors think important when they make the decision. In scenario $Q \in \mathcal{Q}$, the risk premium and the return covariance of the risky assets change and they are denoted by $\hat{\mu}$ and $\hat{V}$, respectively. For a square matrix $M$, let $\operatorname{tr}(M),|M|$ and $M^{\prime}$ be the trace, the determinant and the transpose of $M$, respectively. We can find the explicit form of $K L(Q, P)$ with the following equation,

$$
K L(Q, P)=\frac{1}{2}\left[\ln |V|-\ln |\hat{V}|+\operatorname{tr}\left(V^{-1} \hat{V}\right)-n+(\mu-\hat{\mu})^{\prime} V^{-1}(\mu-\hat{\mu})\right] .
$$

We now apply the uncertainty model on our asset allocation problem. The investors realize that the distribution of the return of the assets can be different from their expectation due to various reasons (estimation errors, distribution changing over time and etc.). Due to their uncertainty aversion, they are more conservative in the real market compared with a market with no uncertainty. The worst scenario in the set of measures is chosen in our analysis. It can illustrate the uncertainty aversion behaviour of the investors. Let $\mathcal{U}$ be the set of parameters $(\hat{\mu}, \hat{V})$ under the measure $Q \in \mathcal{Q}$. In this section, we assume that $V$ is fixed in the set $\mathcal{U}$ and use $\mathcal{U}_{\mu}$ to denote the corresponding feasible set of parameters. Using the mean-variance approach, if $r_{p}$ is the required risk premium of the portfolio in the worst scenario which 
is non-negative, $u$ is the composition of the portfolio, the asset allocation problem can be formulated as

$$
\min _{u \in \mathbf{R}^{n}} u^{\prime} V u
$$

such that $\quad u^{\prime} \hat{\mu} \geq r_{p}$ for all $(\hat{\mu}, V) \in \mathcal{U}_{\mu}$ and $K L(Q, P) \leq K$.

As $V$ is fixed, $\hat{\mu}$ is the only source of uncertainty. The relative entropy function can be simplified as

$$
K L(Q, P)=\frac{1}{2}\left[(\mu-\hat{\mu})^{\prime} V^{-1}(\mu-\hat{\mu})\right]
$$

Applying the theory of multi-objective optimization, the original constrained optimization problem can be modified and it becomes

$$
\max _{u} \min _{\hat{\mu}} \quad u^{\prime} \hat{\mu}-\lambda u^{\prime} V u+\frac{\gamma}{2}(\mu-\hat{\mu})^{\prime} V^{-1}(\mu-\hat{\mu}) .
$$

where $\lambda, \gamma>0$. The relative entropy function is convex and differentiable with respect to the model parameters. Karush-Kuhn-Tucker conditions are satisfied and the Lagrangian (4) has the same solution as the original problem. For more details of the convex optimization and the Lagrangian approach, we can refer to Boyd and Vandenberghe (2004). Let

$$
f_{1}(u, \hat{\mu}):=u^{\prime} \hat{\mu}-\lambda u^{\prime} V u+\frac{\gamma}{2}(\mu-\hat{\mu})^{\prime} V^{-1}(\mu-\hat{\mu}) .
$$

Differentiate $f_{1}(u, \hat{\mu})$ with respect to $u$ and $\hat{\mu}$ and obtain

$$
\frac{\partial f_{1}(u, \hat{\mu})}{\partial u^{\prime}}=\hat{\mu}-2 \lambda V u, \quad \frac{\partial f_{1}(u, \hat{\mu})}{\partial \hat{\mu}}=u^{\prime}-\gamma(\mu-\hat{\mu})^{\prime} V^{-1}
$$

The optimal investment strategy $u^{*}$ and the worst scenario premium $\mu^{*}$ are

$$
u^{*}=\frac{1}{2 \lambda} V^{-1} \mu^{*}, \quad \mu^{*}=\frac{2 \lambda \gamma}{1+2 \lambda \gamma} \mu .
$$

Next, we find $\lambda$ and $\gamma$ and solve the problem. Let $\widetilde{Q}$ be the risk neutral measure, $\widetilde{K}$ be the relative entropy of $\widetilde{Q}$ with respect to $P$. We have $\widetilde{K}:=$ $K L(\underset{\widetilde{Q}}{\widetilde{K}} P)=\frac{1}{2} \mu^{\prime} V^{-1} \mu$. We solve the problem in two cases, $K<\widetilde{K}$ and $K \geq \widetilde{K}$. 
(i) If $K<\widetilde{K}$, with the constraints, we obtain

$$
\lambda=\frac{1}{r_{p}}(\sqrt{\widetilde{K}}-\sqrt{K})^{2}, \quad \gamma=\frac{r_{p}}{2 \sqrt{K}(\sqrt{\widetilde{K}}-\sqrt{K})} .
$$

and

$$
u^{*}=\frac{r_{p}}{2 \sqrt{\widetilde{K}}(\sqrt{\widetilde{K}}-\sqrt{K})} V^{-1} \mu, \quad \mu^{*}=\frac{\sqrt{\widetilde{K}}-\sqrt{K}}{\sqrt{\widetilde{K}}} \mu .
$$

(ii) If $K \geq \widetilde{K}$, this indicates $\widetilde{Q} \in \mathcal{Q}$. The risk premium derived from the risky assets can be non-positive in the worst scenario. The optimal strategy $u^{*}$ must be 0 in order to minimize the variance of portfolio and the corresponding worst scenario measure is $\widetilde{Q}$. We can also study the problem by removing the uncertainty constraint. The problem becomes

$$
\max _{u} \min _{\hat{\mu}} \quad u^{\prime} \hat{\mu}-\lambda u^{\prime} V u
$$

Let $f_{2}(u, \hat{\mu}):=u^{\prime} \hat{\mu}-\lambda u^{\prime} V u$. Differentiate $f_{2}(u, \hat{\mu})$ with respect to $u$,

$$
\frac{\partial f_{2}(u, \hat{\mu})}{\partial u^{\prime}}=\hat{\mu}-2 \lambda V u \Rightarrow u^{*}=\frac{1}{2 \lambda} V^{-1} \hat{\mu}
$$

therefore,

$$
f_{2}\left(u^{*}, \hat{\mu}\right)=\frac{1}{4 \lambda} \hat{\mu}^{\prime} V^{-1} \hat{\mu} \Rightarrow \mu^{*}=0 \Rightarrow Q^{*}=\widetilde{Q} .
$$

The risk premium $r_{p}$ is 0 or otherwise there is no solution.

The investors make their investment decision according to the worst scenario in a set of measures. If, in the worst scenario, the risky assets give a worse result than the risk-free asset, the investors avoid buying or short selling any of these risky assets and just invest in the risk-free asset. This is what we observe in the real market. Many people do not invest in risky securities but put all their money in a saving account because they realize the assets' return uncertainty. They might understand the concept of risk premium or the idea of higher risk higher expected return. However, they still choose not to invest in risky assets. They are very conservative. Under this model, we can say that they consider a set of measures which includes the risk neutral one (that is, $K \geq \widetilde{K}$ ). 
For a non-trivial case where $K<\widetilde{K}$, the risk premium of the portfolio under measure $P$ is

$$
u^{* \prime} \mu=\frac{r_{p} \sqrt{\widetilde{K}}}{\sqrt{\widetilde{K}}-\sqrt{K}}=2 \sqrt{K \widetilde{K}} \gamma
$$

and the variance of the portfolio return under measure $P$ is

$$
u^{* \prime} V u^{*}=\frac{r_{p}^{2}}{4 \widetilde{K}(\sqrt{\widetilde{K}}-\sqrt{K})^{2}} \mu^{\prime} V^{-1} \mu=\frac{r_{p}^{2}}{2(\sqrt{\widetilde{K}}-\sqrt{K})^{2}}=\frac{r_{p}}{2 \lambda} .
$$

\section{Uncertainty on Covariances}

In the previous section, we study the effect of uncertainty on the expected return (or, risky premium) of the assets. The result is intuitive and the worst scenario risk premia are a proportional reduction of the best estimated risk premia. Here, we shall study the effect of uncertainty on the covariance matrix $V$ and identify its form in the worst scenario. The effect and the interaction of risk premium and covariance uncertainties will be discussed in the next section.

Assume that $\mu$ is fixed under the set of uncertainty measures. We use $\mathcal{U}_{V}$ to denote the corresponding set of parameters. If $r_{p}$ is the required risk premium in the worst scenario, the asset allocation problem is now

$$
\begin{aligned}
\min _{u \in \mathbf{R}^{n}} \max _{(\mu, \hat{V}) \in \mathcal{U}_{V}} & u^{\prime} \hat{V} u \\
\text { such that } & u^{\prime} \mu \geq r_{p} \text { and } K L(Q, P) \leq K .
\end{aligned}
$$

Since $\mu$ is fixed,

$$
K L(Q, P)=\frac{1}{2}\left[\ln |V|-\ln |\hat{V}|+\operatorname{tr}\left(V^{-1} \hat{V}\right)-n\right] .
$$

Again, we modify the problem and it becomes

$$
\max _{u} \min _{\hat{V}} \quad u^{\prime} \mu-\lambda u^{\prime} \hat{V} u+\frac{\gamma}{2}\left(-\ln |\hat{V}|+\operatorname{tr}\left(V^{-1} \hat{V}\right)\right) .
$$

for some $\lambda, \gamma>0$. Let

$$
f_{3}(u, \hat{V}):=u^{\prime} \mu-\lambda u^{\prime} \hat{V} u+\frac{\gamma}{2}\left(-\ln |\hat{V}|+\operatorname{tr}\left(V^{-1} \hat{V}\right)\right) .
$$


Differentiate $f_{3}(u, \hat{V})$ with respect to $u$ and $\hat{V}$, we obtain

$$
\frac{\partial f_{3}(u, \hat{V})}{\partial u^{\prime}}=\mu-2 \lambda \hat{V} u, \quad \frac{\partial f_{3}(u, \hat{V})}{\partial \hat{V}^{\prime}}=-\lambda u u^{\prime}+\frac{\gamma}{2}\left(V^{-1}-\hat{V}^{-1}\right) .
$$

Therefore

$$
\begin{aligned}
& u^{*}=\frac{1}{2 \lambda} V^{*-1} \mu \quad \text { and } \quad u^{*} u^{* \prime}=\frac{\gamma}{2 \lambda}\left(V^{-1}-V^{*-1}\right) \\
\Rightarrow \quad & V^{*-1} \mu \mu^{\prime} V^{*-1}=2 \lambda \gamma\left(V^{-1}-V^{*-1}\right) .
\end{aligned}
$$

We know that $\hat{V} \succeq 0$ for all feasible $\hat{V} \in \mathcal{U}_{V}$. In fact, we can show that $\hat{V} \succ 0$ for all $\hat{V} \in \mathcal{U}_{V}$ and it will be discussed in Section 4. For a positive definite matrix $V$, there exists a unique square root matrix $M$ such that $M \succ 0$ and $M M=V$. Denote the square root matrix of $V$ by $V^{1 / 2}$. Similarly, we have $V^{-1 / 2} V^{-1 / 2}=V^{-1}$. Let $I$ be the identity matrix. By the symmetry property of covariance matrices,

$$
\begin{aligned}
V^{*} V^{-1} V^{*}-V^{*} & =\frac{1}{2 \lambda \gamma} \mu \mu^{\prime} \\
\left(V^{*}-\frac{1}{2} V\right) V^{-1}\left(V^{*}-\frac{1}{2} V\right) & =\frac{1}{2 \lambda \gamma} \mu \mu^{\prime}+\frac{1}{4} V \\
V^{*}-\frac{1}{2} V & =V^{1 / 2}\left[V^{-1 / 2}\left(\frac{1}{2 \lambda \gamma} \mu \mu^{\prime}+\frac{1}{4} V\right) V^{-1 / 2}\right]^{1 / 2} V^{1 / 2} \\
V^{*} & =\frac{1}{2} V+V^{1 / 2}\left(\frac{1}{4} I+\frac{1}{2 \lambda \gamma} V^{-1 / 2} \mu \mu^{\prime} V^{-1 / 2}\right)^{1 / 2} V^{1 / 2} \\
& =\frac{1}{2} V^{1 / 2}\left[I+\left(I+\frac{2}{\lambda \gamma} V^{-1 / 2} \mu \mu^{\prime} V^{-1 / 2}\right)^{1 / 2}\right] V^{1 / 2} .
\end{aligned}
$$

As $\mu \mu^{\prime} \succeq 0$, all the above square root matrices exist. Also, $V^{-1 / 2} \mu \mu^{\prime} V^{-1 / 2}$ is symmetric, by Eigen-decomposition, we have

$$
V^{-1 / 2} \mu \mu^{\prime} V^{-1 / 2}=G D G^{\prime}
$$

$D$ is a diagonal matrix with the eigenvalues of $V^{-1 / 2} \mu \mu^{\prime} V^{-1 / 2}$ as its principal diagonal entries. $G$ is an orthogonal matrix with the corresponding eigenvectors as its columns. Without loss of generality, we take the first diagonal 
entry of $D$ as $\mu^{\prime} V^{-1} \mu$ (the others are zero) and the first column of $G$ is $V^{-1 / 2} \mu / \sqrt{\mu^{\prime} V^{-1} \mu}$. That is,

$$
D=\left(\begin{array}{ccc}
2 \widetilde{K} & & \\
& 0 & \\
& & \ddots
\end{array}\right) \quad \text { and } \quad G=\left(\begin{array}{ccc}
\frac{V^{-1 / 2} \mu}{\sqrt{2 \widetilde{K}}} & \vdots & \vdots
\end{array}\right) \text {. }
$$

We have

$$
\left(I+\frac{2}{\lambda \gamma} V^{-1 / 2} \mu \mu^{\prime} V^{-1 / 2}\right)^{1 / 2}=G\left(I+\frac{2}{\lambda \gamma} D\right)^{1 / 2} G^{\prime} .
$$

Therefore,

$$
\begin{aligned}
V^{*} & =\frac{1}{2} V^{1 / 2}\left[I+G\left(I+\frac{2}{\lambda \gamma} D\right)^{1 / 2} G^{\prime}\right] V^{1 / 2} \\
& =V^{1 / 2} G\left[\frac{1}{2}\left(I+\left(I+\frac{2}{\lambda \gamma} D\right)^{1 / 2}\right)\right] G^{\prime} V^{1 / 2} \\
\ln \left|V^{*}\right| & =\ln \left|V^{1 / 2} G\left[\frac{1}{2}\left(I+\left(I+\frac{2}{\lambda \gamma} D\right)^{1 / 2}\right)\right] G^{\prime} V^{1 / 2}\right| \\
& =\ln |V|+\ln \left|\frac{1}{2}\left(I+\left(I+\frac{2}{\lambda \gamma} D\right)^{1 / 2}\right)\right| \\
& =\ln |V|+\ln \left(\frac{1}{2}+\left(\frac{1}{4}+\frac{\widetilde{K}}{\lambda \gamma}\right)^{1 / 2}\right) .
\end{aligned}
$$

Moreover, by the cyclic property of trace,

$$
\begin{aligned}
\operatorname{tr}\left(V^{-1} V^{*}\right) & =\operatorname{tr}\left(V^{-1 / 2} V^{-1 / 2} V^{*}\right)=\operatorname{tr}\left(V^{-1 / 2} V^{*} V^{-1 / 2}\right) \\
& =\operatorname{tr}\left[G\left[\frac{1}{2}\left(I+\left(I+\frac{2}{\lambda \gamma} D\right)^{1 / 2}\right)\right] G^{\prime}\right] \\
& =\frac{1}{2} \operatorname{tr}\left(I+\left(I+\frac{2}{\lambda \gamma} D\right)^{1 / 2}\right) \\
& =n-\frac{1}{2}+\left(\frac{1}{4}+\frac{\widetilde{K}}{\lambda \gamma}\right)^{1 / 2} .
\end{aligned}
$$


The relative entropy of $Q^{*}$ with respect to $P$ is

$$
\frac{1}{2}\left[-\ln \left(\frac{1}{2}+\left(\frac{1}{4}+\frac{\widetilde{K}}{\lambda \gamma}\right)^{1 / 2}\right)-\frac{1}{2}+\left(\frac{1}{4}+\frac{\widetilde{K}}{\lambda \gamma}\right)^{1 / 2}\right]
$$

Inferred from the form of the relative entropy, define $\theta:=\frac{1}{2}+\left(\frac{1}{4}+\frac{\widetilde{K}}{\lambda \gamma}\right)^{1 / 2}$ and $g_{1}(\theta):=\frac{1}{2}(\theta-1-\ln \theta)$ for $\theta \geq 1$. We can check that $g_{1}$ is a strictly increasing function of $\theta$ for $\theta>1$ and so $g_{1}^{-1}$ exists. Using the uncertainty constraint, we can find $\theta$,

$$
g_{1}(\theta)=K \Rightarrow \theta=\frac{1}{2}+\left(\frac{1}{4}+\frac{\widetilde{K}}{\lambda \gamma}\right)^{1 / 2}=g_{1}^{-1}(K)
$$

We find $\theta$ as a function of $K$, and it can be used to find the other unknowns in the problem. With the expected return constraint,

$$
u^{* \prime} \mu=\frac{1}{2 \lambda} \mu^{\prime} V^{*-1} \mu=r_{p}
$$

$\lambda$ is given by,

$$
\begin{aligned}
& \lambda=\frac{\mu^{\prime} V^{*-1} \mu}{2 r_{p}} \\
& =\frac{1}{2 r_{p}} \mu^{\prime} V^{-1 / 2} G\left[\frac{1}{2}\left(I+\left(I+\frac{2}{\lambda \gamma} D\right)^{1 / 2}\right)\right]^{-1} G^{\prime} V^{-1 / 2} \mu \\
& =\frac{1}{2 r_{p}} \mu^{\prime} V^{-1 / 2} G\left(\begin{array}{llll}
\frac{1}{2}+\left(\frac{1}{4}+\frac{\widetilde{K}}{\lambda \gamma}\right)^{1 / 2} & & & \\
& 1 & & \\
& & \ddots
\end{array}\right)^{-1} G^{\prime} V^{-1 / 2} \mu \\
& =\frac{1}{2 r_{p}}\left(\begin{array}{c}
\sqrt{\mu^{\prime} V^{-1} \mu} \\
0 \\
0
\end{array}\right)^{\prime}\left(\begin{array}{ccc}
\theta^{-1} & & \\
& 1 & \\
& & \ddots
\end{array}\right)\left(\begin{array}{c}
\sqrt{\mu^{\prime} V^{-1} \mu} \\
0 \\
0
\end{array}\right) \\
& =\frac{\widetilde{K}}{\theta r_{p}} \text {. }
\end{aligned}
$$


Using equation (8), we have

$$
\frac{\widetilde{K}}{\lambda \gamma}=\left(\theta-\frac{1}{2}\right)^{2}-\frac{1}{4}, \quad \lambda \gamma=\frac{\widetilde{K}}{\left(\theta-\frac{1}{2}\right)^{2}-\frac{1}{4}} .
$$

Then, we can obtain $\gamma$,

$$
\gamma=\frac{\widetilde{K}}{\lambda\left(\left(\theta-\frac{1}{2}\right)^{2}-\frac{1}{4}\right)}=\frac{\theta r_{p}}{\left(\theta-\frac{1}{2}\right)^{2}-\frac{1}{4}} .
$$

Similarly, we can find $V^{*}$ using the characteristic of $G$,

$$
\begin{aligned}
V^{*} & =V^{1 / 2} G\left[\frac{1}{2}\left(I+\left(I+\frac{2}{\lambda \gamma} D\right)^{1 / 2}\right)\right] G^{\prime} V^{1 / 2} \\
& =V^{1 / 2} G\left(\begin{array}{rrr}
\theta & & \\
& 1 & \\
& & \ddots
\end{array}\right) G^{\prime} V^{1 / 2} \\
& =V+\frac{\theta-1}{2 \widetilde{K}} \mu \mu^{\prime} .
\end{aligned}
$$

The optimal strategy $u^{*}$ is

$$
\begin{aligned}
u^{*} & =\frac{1}{2 \lambda} V^{*-1} \mu \\
& =\frac{\theta r_{p}}{2 \widetilde{K}} V^{-1 / 2}\left(I+\frac{\theta^{-1}-1}{2 \widetilde{K}} V^{-1 / 2} \mu \mu^{\prime} V^{-1 / 2}\right) V^{-1 / 2} \mu \\
& =\frac{\theta r_{p}}{2 \widetilde{K}}\left(V^{-1} \mu+\frac{\theta^{-1}-1}{2 \widetilde{K}} V^{-1} \mu\left(\mu^{\prime} V^{-1} \mu\right)\right) \\
& =\frac{r_{p}}{2 \widetilde{K}} V^{-1} \mu .
\end{aligned}
$$

Risk premium uncertainty achieves its maximum effect when the uncertainty parameter equals $\widetilde{K}$. Due to the use of optimal investment strategy and the presence of the risk-free asset, the minimum possible risk premium is zero. For the covariance uncertainty, its effect increases with the uncertainty parameters without limit. 
The effect of covariance uncertainty can be illustrated by calculating the variance of the return of the optimal portfolio. If the target risk premium under measure $Q^{*}$ is $r_{p}$, under measure $P$, the variance is

$$
u^{* \prime} V u^{*}=\frac{r_{p}^{2}}{4 \widetilde{K}^{2}} \mu^{\prime} V^{-1} \mu=\frac{r_{p}^{2}}{2 \widetilde{K}},
$$

while the worst scenario variance is

$$
u^{* \prime} V^{*} u^{*}=\frac{r_{p}^{2}}{4 \widetilde{K}^{2}} \mu^{\prime} V^{-1}\left(V+\frac{\theta-1}{2 \widetilde{K}} \mu \mu^{\prime}\right) V^{-1} \mu=\frac{\theta r_{p}^{2}}{2 \widetilde{K}} .
$$

The increase in variance due to covariance uncertainty is $(\theta-1) r_{p}^{2} /(2 \widetilde{K})$.

\section{Uncertainty on Risk Premia, Covariances and Further Analysis}

We have discussed the effects of risk premium uncertainty and covariance uncertainty separately and recognize their differences in their corresponding worst scenarios. They will be studied under the same framework in this section. Consider

$$
\begin{aligned}
\min _{u \in \mathbf{R}^{n}} \max _{(\hat{\mu}, \hat{V}) \in \mathcal{U}} & u^{\prime} \hat{V} u \\
\text { such that } & u^{\prime} \hat{\mu} \geq r_{p} \text { and } K L(Q, P) \leq K,
\end{aligned}
$$

where

$$
K L(Q, P)=\frac{1}{2}\left[\ln |V|-\ln |\hat{V}|+\operatorname{tr}\left(V^{-1} \hat{V}\right)-n+(\mu-\hat{\mu})^{\prime} V^{-1}(\mu-\hat{\mu})\right] .
$$

In fact, if we want to ensure that there exists a unique solution of these problems, we have to show that the uncertainty set $\mathcal{U}$ is convex. Covariance matrix $V$ under measure $P$ is positive definite. However, $\hat{V}$ under measure $Q \in \mathcal{Q}$ might have a determinant equal to zero. We know $\hat{V} \succeq 0$, which must be true for covariance matrix. Notice that $K L(Q, P) \rightarrow+\infty$ when $|\hat{V}| \rightarrow 0$. By defining $K L(Q, P)=+\infty$ when $|\hat{V}|=0, \hat{V} \succ 0$ if $(\hat{\mu}, \hat{V}) \in \mathcal{U}$ for a bounded $K .|\hat{V}|>\epsilon$ for some positive $\epsilon$ and $\hat{V}^{-1}$ exists. 
For $\left(\mu_{1}, V_{1}\right),\left(\mu_{2}, V_{2}\right) \in \mathcal{U}$, we want to see whether $\left(\left(\mu_{1}+\mu_{2}\right) / 2,\left(V_{1}+V_{2}\right) / 2\right)$ is in $\mathcal{U}$. By Cauchy-Schwartz,

$$
\begin{aligned}
& \left(\frac{\mu_{1}+\mu_{2}}{2}-\mu\right)^{\prime} V^{-1}\left(\frac{\mu_{1}+\mu_{2}}{2}-\mu\right) \\
= & \frac{1}{4}\left[\left(\mu_{1}-\mu\right)^{\prime} V^{-1}\left(\mu_{1}-\mu\right)+\left(\mu_{2}-\mu\right)^{\prime} V^{-1}\left(\mu_{2}-\mu\right)+2\left(\mu_{1}-\mu\right)^{\prime} V^{-1}\left(\mu_{2}-\mu\right)\right] \\
\leq & \frac{1}{4}\left[\left(\mu_{1}-\mu\right)^{\prime} V^{-1}\left(\mu_{1}-\mu\right)+\left(\mu_{2}-\mu\right)^{\prime} V^{-1}\left(\mu_{2}-\mu\right)\right. \\
& \left.\quad+2\left[\left(\mu_{1}-\mu\right)^{\prime} V^{-1}\left(\mu_{1}-\mu\right) \cdot\left(\mu_{2}-\mu\right)^{\prime} V^{-1}\left(\mu_{2}-\mu\right)\right]^{1 / 2}\right] \\
\leq & \frac{1}{2}\left[\left(\mu_{1}-\mu\right)^{\prime} V^{-1}\left(\mu_{1}-\mu\right)+\left(\mu_{2}-\mu\right)^{\prime} V^{-1}\left(\mu_{2}-\mu\right)\right] .
\end{aligned}
$$

For the variance part on the relative entropy, by Lawson and Lim (2001),

$$
\frac{1}{2}\left(V_{1}+V_{2}\right) \succeq V_{1}^{1 / 2}\left(V_{1}^{-1 / 2} V_{2} V_{1}^{-1 / 2}\right)^{1 / 2} V_{1}^{1 / 2} .
$$

By Theorem 7.8 of Zhang (2011),

$$
\begin{aligned}
\left|\frac{1}{2}\left(V_{1}+V_{2}\right)\right| & \geq\left|V_{1}\left(V_{1}^{-1 / 2} V_{2} V_{1}^{-1 / 2}\right)^{1 / 2} V_{1}^{1 / 2}\right| \\
& =\left|V_{1}\right|\left|V_{1}^{-1 / 2}\right|^{1 / 2}\left|V_{2}\right|^{1 / 2}\left|V_{1}^{-1 / 2}\right|^{1 / 2} \\
& =\left|V_{1}\right|^{1 / 2}\left|V_{2}\right|^{1 / 2}>0 \\
\Rightarrow \ln \left|\frac{1}{2}\left(V_{1}+V_{2}\right)\right| & \geq \frac{1}{2}\left(\ln \left|V_{1}\right|+\ln \left|V_{2}\right|\right) .
\end{aligned}
$$

For $\alpha \in(0,1)$,

$$
V_{1}, V_{2} \succ 0 \Rightarrow \alpha V_{1}+(1-\alpha) V_{2} \succ 0 .
$$

Therefore, $K L$ is a convex function of $(\hat{\mu}, \hat{V})$ and $\mathcal{U}$ is a convex set. The same result can be applied in the previous two sections.

Using Lagrangian method, the problem becomes

$$
\max _{u} \min _{(\hat{\mu}, \hat{V})} u^{\prime} \hat{\mu}-\lambda u^{\prime} \hat{V} u+\frac{\gamma}{2}\left((\mu-\hat{\mu})^{\prime} V^{-1}(\mu-\hat{\mu})-\ln |\hat{V}|+\operatorname{tr}\left(V^{-1} \hat{V}\right)\right)
$$


for some $\lambda, \gamma>0$. Let

$f_{4}(u, \hat{\mu}, \hat{V}):=u^{\prime} \hat{\mu}-\lambda u^{\prime} \hat{V} u+\frac{\gamma}{2}\left((\mu-\hat{\mu})^{\prime} V^{-1}(\mu-\hat{\mu})-\ln |\hat{V}|+\operatorname{tr}\left(V^{-1} \hat{V}\right)\right)$.

Differentiate $f_{4}(u, \hat{\mu}, \hat{V})$ with respect to $u, \hat{\mu}$ and $\hat{V}$,

$$
\begin{gathered}
\frac{f_{4}(u, \hat{\mu}, \hat{V})}{\partial u^{\prime}}=\hat{\mu}-2 \lambda \hat{V} u, \quad \frac{f_{4}(u, \hat{\mu}, \hat{V})}{\partial \hat{\mu}}=u^{\prime}-\gamma(\mu-\hat{\mu})^{\prime} V^{-1}, \\
\frac{f_{4}(u, \hat{\mu}, \hat{V})}{\partial \hat{V}^{\prime}}=-\lambda u u^{\prime}+\frac{\gamma}{2}\left(V^{-1}-\hat{V}^{-1}\right) .
\end{gathered}
$$

Using equation (14),

$$
\begin{aligned}
u^{*}=\frac{1}{2 \lambda} V^{*-1} \mu^{*} & =\gamma V^{-1}\left(\mu-\mu^{*}\right) \\
V^{*-1} \mu^{*} & =2 \lambda \gamma V^{-1}\left(\mu-\mu^{*}\right) .
\end{aligned}
$$

Together with equation (15), we have

$$
\begin{aligned}
\frac{\gamma}{2}\left(V^{-1}-V^{*-1}\right) & =\lambda \gamma^{2} V^{-1}\left(\mu-\mu^{*}\right)\left(\mu-\mu^{*}\right)^{\prime} V^{-1} \\
V^{*-1} & =V^{-1}-2 \lambda \gamma V^{-1}\left(\mu-\mu^{*}\right)\left(\mu-\mu^{*}\right)^{\prime} V^{-1} \\
V^{*-1} \mu^{*} & =V^{-1} \mu^{*}-2 \lambda \gamma V^{-1}\left(\mu-\mu^{*}\right)\left(\mu-\mu^{*}\right)^{\prime} V^{-1} \mu^{*} \\
\Rightarrow 2 \lambda \gamma V^{-1}\left(\mu-\mu^{*}\right) & =V^{-1} \mu^{*}-2 \lambda \gamma V^{-1}\left(\mu-\mu^{*}\right)\left(\mu-\mu^{*}\right)^{\prime} V^{-1} \mu^{*} .
\end{aligned}
$$

Therefore,

$$
\begin{aligned}
2 \lambda \gamma\left(\mu-\mu^{*}\right) & =\mu^{*}-2 \lambda \gamma\left(\mu-\mu^{*}\right)\left(\mu-\mu^{*}\right)^{\prime} V^{-1} \mu^{*} \\
\mu^{*} & =\frac{2 \lambda \gamma\left(1+\left(\mu-\mu^{*}\right)^{\prime} V^{-1} \mu^{*}\right)}{2 \lambda \gamma\left(1+\left(\mu-\mu^{*}\right)^{\prime} V^{-1} \mu^{*}\right)+1} \mu:=\zeta \mu,
\end{aligned}
$$

for $\zeta \in(0,1)$. So,

$$
\left(\mu-\mu^{*}\right)^{\prime} V^{-1} \mu^{*}=\zeta(1-\zeta) \mu^{\prime} V^{-1} \mu=2 \zeta(1-\zeta) \widetilde{K}
$$

With the definition of $\zeta$,

$$
\begin{aligned}
2 \lambda \gamma\left(1+\left(\mu-\mu^{*}\right)^{\prime} V^{-1} \mu^{*}\right) & =\left(2 \lambda \gamma\left(1+\left(\mu-\mu^{*}\right)^{\prime} V^{-1} \mu^{*}\right)+1\right) \zeta \\
\zeta & =2 \lambda \gamma\left(1+\left(\mu-\mu^{*}\right)^{\prime} V^{-1} \mu^{*}\right)(1-\zeta) \\
& =2 \lambda \gamma(1+2 \zeta(1-\zeta) \widetilde{K})(1-\zeta) \\
\lambda \gamma & =\frac{\zeta}{2(1+2 \zeta(1-\zeta) \widetilde{K})(1-\zeta)}
\end{aligned}
$$


Now, we can obtain an equation for $\zeta$,

$$
4 \lambda \gamma \widetilde{K} \zeta^{3}-8 \lambda \gamma \widetilde{K} \zeta^{2}+(4 \lambda \gamma \widetilde{K}-2 \lambda \gamma-1) \zeta+2 \lambda \gamma=0 .
$$

Let $g_{2}(x ; \lambda, \gamma):=4 \lambda \gamma \widetilde{K} x^{3}-8 \lambda \gamma \widetilde{K} x^{2}+(4 \lambda \gamma \widetilde{K}-2 \lambda \gamma-1) x+2 \lambda \gamma$. We know that $\lambda \gamma>0, g_{2}(0)=2 \lambda \gamma>0$, and $g_{2}(1)=-1<0$. Thus, for this cubic equation, exactly one root is greater than 1 and exactly one root is smaller than 0 . There exists a unique solution for $g_{2}(\zeta)=0$ where $\zeta \in(0,1)$. Now, we try to find $\lambda$ and $\gamma$ in terms of $\zeta$. We find the worst scenario covariance matrix,

$$
\begin{aligned}
V^{*-1} & =V^{-1}-2 \lambda \gamma V^{-1}\left(\mu-\mu^{*}\right)\left(\mu-\mu^{*}\right)^{\prime} V^{-1} \\
& =V^{-1 / 2}\left(I-2 \lambda \gamma V^{-1 / 2}\left(\mu-\mu^{*}\right)\left(\mu-\mu^{*}\right)^{\prime} V^{-1 / 2}\right) V^{-1 / 2} \\
& =V^{-1 / 2}\left(I-2 \lambda \gamma(1-\zeta)^{2} V^{-1 / 2} \mu \mu^{\prime} V^{-1 / 2}\right) V^{-1 / 2} \\
& =V^{-1 / 2} G\left(\begin{array}{rrr}
1-4 \lambda \gamma(1-\zeta)^{2} \widetilde{K} & & \\
& 1 & \\
V^{*} & = & V^{1 / 2} G\left(\begin{array}{rrr}
\left(1-4 \lambda \gamma(1-\zeta)^{2} \widetilde{K}\right)^{-1} & & \ddots
\end{array}\right) G^{\prime} V^{-1 / 2} \\
& & 1
\end{array}\right) G^{\prime} V^{1 / 2} .
\end{aligned}
$$

Using equation (16), we know

$$
\begin{aligned}
4 \lambda \gamma(1-\zeta)^{2} \widetilde{K} & =\frac{2 \zeta(1-\zeta) \widetilde{K}}{1+2 \zeta(1-\zeta) \widetilde{K}}, \\
1-4 \lambda \gamma(1-\zeta)^{2} \widetilde{K} & =(1+2 \zeta(1-\zeta) \widetilde{K})^{-1} .
\end{aligned}
$$

For the uncertainty constraint, we have

$$
\begin{aligned}
\ln |V|-\ln \left|V^{*}\right| & =-\ln (1+2 \zeta(1-\zeta) \widetilde{K}), \\
\operatorname{tr}\left(V^{-1} V^{*}\right) & =\operatorname{tr}\left(V^{-1 / 2} V^{*} V^{-1 / 2}\right)=n+2 \zeta(1-\zeta) \widetilde{K}, \\
\left(\mu-\mu^{*}\right)^{\prime} V^{-1}\left(\mu-\mu^{*}\right) & =2(1-\zeta)^{2} \widetilde{K} .
\end{aligned}
$$

Hence,

$$
\begin{aligned}
\frac{1}{2}\left[-\ln (1+2 \zeta(1-\zeta) \widetilde{K})+2 \zeta(1-\zeta)+2(1-\zeta)^{2} \widetilde{K}\right] & =K \\
\frac{-1}{2} \ln (1+2 \zeta(1-\zeta) \widetilde{K})+(1-\zeta) \widetilde{K} & =K
\end{aligned}
$$


Let $g_{3}(\zeta):=\frac{-1}{2} \ln (1+2 \zeta(1-\zeta) \widetilde{K})+(1-\zeta) \widetilde{K}$ for $\zeta \in[0,1] . g_{3}(0)=\widetilde{K}$ and $g_{3}(1)=0$. Furthermore,

$$
\begin{aligned}
\frac{d g_{3}(\zeta)}{d \zeta} & =\frac{-(2-4 \zeta) \widetilde{K}}{2(1+2 \zeta(1-\zeta) \widetilde{K})}-\widetilde{K} \\
& =\frac{-2(1-\zeta)(1+\zeta \widetilde{K})}{1+2 \zeta(1-\zeta) \widetilde{K}} \widetilde{K}<0, \quad \zeta \in(0,1) .
\end{aligned}
$$

Therefore, $g_{3}^{-1}(K)$ exists for $K \in[0, \widetilde{K}]$ and $\zeta=g_{3}^{-1}(K)$.

We recognize that the problem can be solved in two cases as in Section 2. For $K \geq \overparen{K}$, the risk neutral probability is within the set of uncertainty measure. Therefore, the best strategy is to make no risky investment as the worst expected return of these risky assets is no better than the risk-free asset. The worst scenario measure is the risk neutral measure.

We now focus on the non-trivial case when $K<\widetilde{K}$. When $K=0$, it is a model with no uncertainty. Then, $\mu^{*}=\mu, V^{*}=V$ and $\zeta=1=g_{3}^{-1}(0)$. For other positive $K$, we can find $\zeta$ using the function $g_{3}^{-1}$ and

$$
\begin{aligned}
V^{*} & =V^{1 / 2} G\left(\begin{array}{ccc}
1+2 \zeta(1-\zeta) \widetilde{K} & & \\
& 1 & \\
& & \ddots
\end{array}\right) G^{\prime} V^{1 / 2} \\
V^{*-1} & =\left(V+\zeta(1-\zeta) \mu \mu^{\prime},\right. \\
& =V^{-1}+V^{-1} \mu \mu^{\prime} V^{-1} \frac{(1+2 \zeta(1-\zeta) \widetilde{K})^{-1}-1}{2 \widetilde{K}} .
\end{aligned}
$$

With the risk premium constraint, we have

$$
\begin{aligned}
\lambda & =\frac{\zeta^{2} \widetilde{K}}{r_{p}(1+2 \zeta(1-\zeta) \widetilde{K})}, \\
\gamma & =\frac{r_{p}}{2 \zeta(1-\zeta) \widetilde{K}}, \\
u^{*} & =\frac{r_{p}}{2 \zeta \widetilde{K}} V^{-1} \mu .
\end{aligned}
$$

For the optimal portfolio, the risk premium is $u^{* \prime} \mu=r_{p} / \zeta$ under measure $P$. Variances of the return under measures $P$ and $Q^{*}$ are $\frac{r_{p}^{2}}{2 \zeta^{2} \widetilde{K}}$ and $\frac{r_{p}^{2}(1+2 \zeta(1-\zeta) \widetilde{K})}{2 \zeta^{2} \widetilde{K}}$, respectively. 
From the result, when uncertainty increases, risk premia reduce. However, we also notice that covariances are not strictly increasing with $K$. With a small uncertainty, when $K$ increases, covariance $V^{*}$ increases. It achieves the maximum when $\zeta=1 / 2$ and $K=K_{1 / 2}:=\frac{\widetilde{K}}{2}-\frac{1}{2} \ln \left(1+\frac{\widetilde{K}}{2}\right)$. When $K$ further increases, $V^{*}$ reduces progressively. It equals $V$ when $K=\widetilde{K}$. It is counter-intuitive and seems to be inconsistent with the meaning of the worst scenario. The result shows that it is more "cost effective" to place more attention on the risk premium uncertainty rather than the covariance uncertainty when we have a high level of conservativeness.

\section{Numerical Examples and Illustrations}

We use a simple example to demonstrate the application of the model and then discuss other implications of the results. Ten-year (Jun 2005 to Jun 2015) monthly return data of 4 major indices (S\&P 500, DAX, HSI and FTSE 100) are used to illustrate our uncertainty approach. The present numerical study is to give us some ideas on the empirical results that we can obtain in practice. It is not a critical review of the validity of the meanvariance model or a study of the security markets. The risk-free rate is taken to be one-year treasury yield $0.28 \%$. Let the covariance matrix and risk premium vector of the 4 assets in an one-year period be

$V=\left(\begin{array}{cccc}0.018632 & 0.020056 & 0.020646 & 0.015213 \\ 0.020056 & 0.034507 & 0.027412 & 0.020652 \\ 0.020646 & 0.027412 & 0.048680 & 0.021663 \\ 0.015213 & 0.020652 & 0.021663 & 0.018791\end{array}\right), \quad \mu=\left(\begin{array}{r}6.1166 \% \\ 10.9547 \% \\ 9.0358 \% \\ 4.0923 \%\end{array}\right)$,

respectively. We can find the relative entropy of the risk neutral measure

$$
\widetilde{K}=\frac{1}{2} \mu^{\prime} V^{-1} \mu=0.238888 \text {. }
$$

It is the maximum effective relative entropy for modelling risk premium uncertainty. If the uncertainty of an investor on the assets is greater than $\widetilde{K}$, it is a trivial case and the investor makes no investment on the risky assets. By using the result in Section 4, we notice that there is a one-to-one corresponding relation between $K$ and $\zeta$ when $K \in[0, \widetilde{K})$. If we are given the value of $K, \zeta$ can be obtained numerically using computer programme with the inverse function we find in the last section. Here, we choose $\zeta$ equal to 


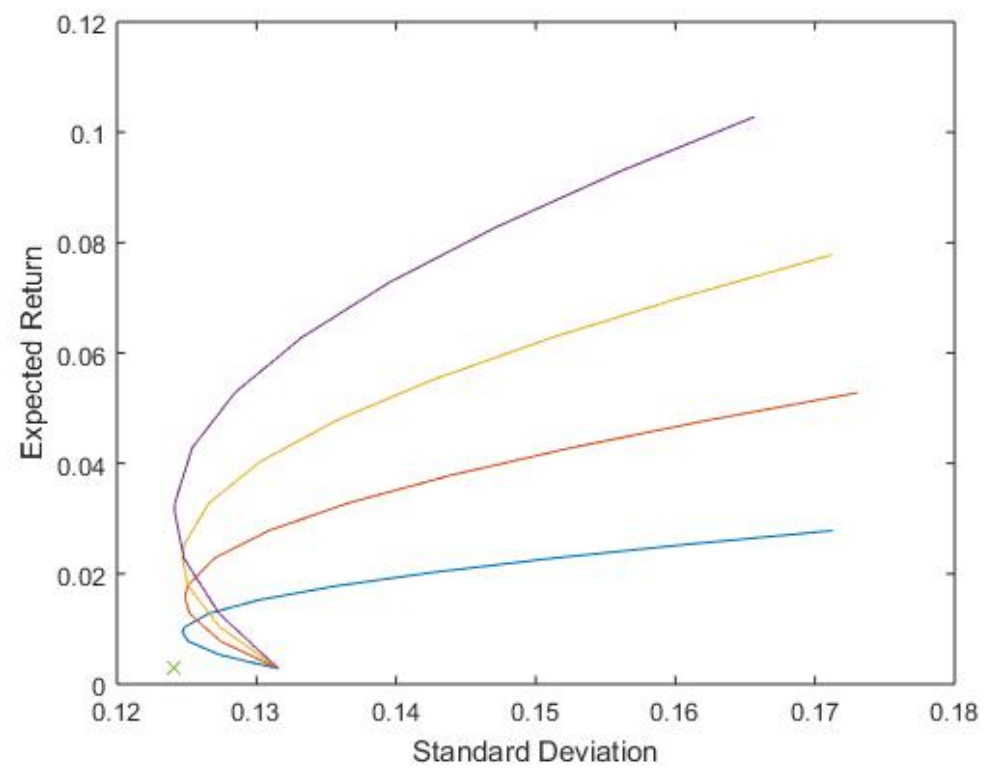

Figure 1: Minimum variance frontiers of the risky assets under different uncertainty levels

$0,0.25,0.5,0.75$ and 1 to illustrate the effect of uncertainty on the minimum variance frontier and the efficient frontier of the portfolios formed by the four indices in the corresponding worst scenarios. Note that $\zeta=0$ might refer to all cases that $K \geq \widetilde{K}$. We specify here that when we say $\zeta=0$ in this section, it is referring to the case that $K=\widetilde{K}$.

In Figure 1, we can see the four minimum variance frontiers under different levels of uncertainty, from the top one for $\zeta=1$ (without uncertainty) to the bottom one for $\zeta=0.25$. When $\zeta=0$, the expected returns of all the four indices are equal to the risk-free rate in the worst scenario. Investor chooses the minimum variance portfolio and it is represented by the small cross at the lower left hand corner in the figure. The minimum variance frontiers are flattened when the uncertainty increases.

Now, we concentrate on the efficient frontiers in Figure 2. The minimum variance portfolios under different levels of uncertainty are joined together by the dashed line. Their standard deviations only change slightly for different $K$. For a given target expected return, the increase of the standard deviation of the efficient portfolios is mainly due to the decrease of risk premia (and the increase in leverage) rather than the change in covariances, especially 


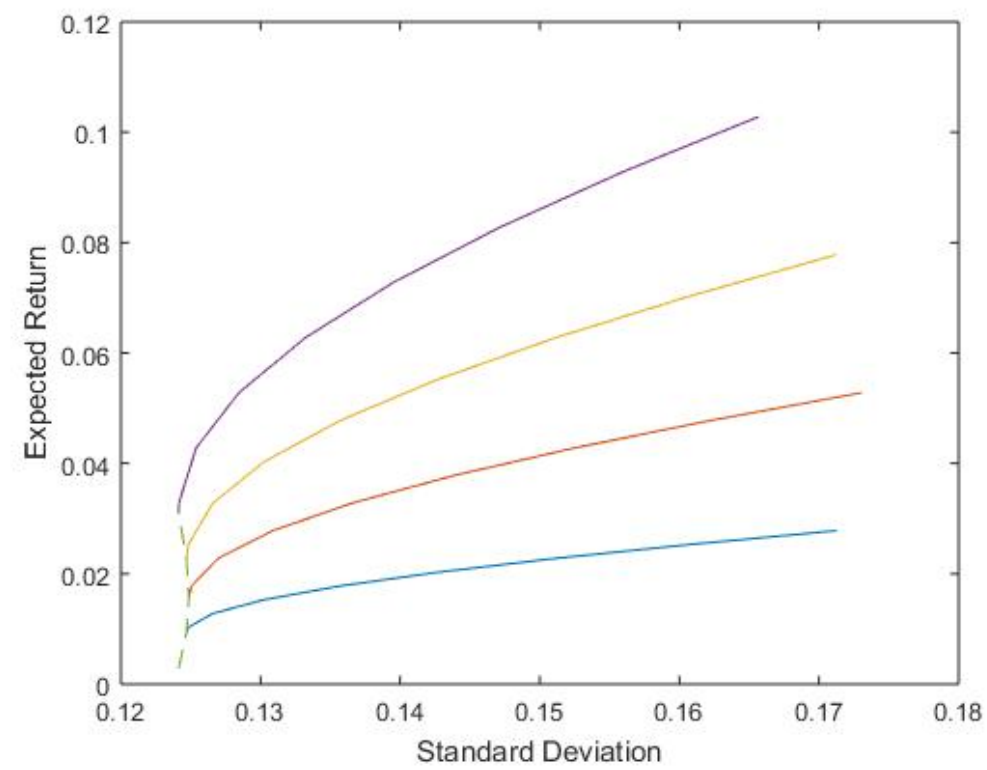

Figure 2: Efficient frontiers of the risky assets under different uncertainty levels

when $\zeta$ is small and the efficient frontier is flat.

It is interesting to note that all the minimum variance frontiers meet when the required expected return equals the risk-free rate. For these minimum variance frontiers, we introduce an additional constraint that all the capital should be invested into these risky assets. Let $\mathbf{1}_{n}$ be the $n$-dimensional column vector $(1,1, \ldots, 1)^{\prime}$. With different $K \in[0, \widetilde{K})$, we have different pairs of $(\hat{\mu}, \hat{V})$. We can find the variance of these minimum variance portfolios by

$$
\max _{u} \quad u^{\prime} \hat{\mu}-\hat{\alpha} u^{\prime} \hat{V} u-\hat{\beta} u^{\prime} \mathbf{1}_{n}
$$

for some $\hat{\alpha}, \hat{\beta}>0$. If the portfolio has zero risk premium, we have constraints $u^{\prime} \hat{\mu}=0$ and $u^{\prime} \mathbf{1}_{n}=1$. Let $f_{5}(u)=u^{\prime} \hat{\mu}-\hat{\alpha} u^{\prime} \hat{V} u-\hat{\beta} u^{\prime} \mathbf{1}_{n}$. Differentiate $f_{5}$ with respect to $u$, we use the constraints and obtain

$\hat{\alpha}=\hat{\mu}^{\prime} \hat{V}^{-1} \mathbf{1}_{n}-\frac{\hat{\mu}^{\prime} \hat{V}^{-1} \hat{\mu}}{\hat{\mu}^{\prime} \hat{V}^{-1} \mathbf{1}_{n}} \mathbf{1}_{n} \hat{V}^{-1} \mathbf{1}_{n}, \quad \hat{\beta}=\frac{\hat{\mu}^{\prime} \hat{V}^{-1} \hat{\mu}}{\hat{\mu}^{\prime} \hat{V}^{-1} \mathbf{1}_{n}}, \quad u^{*}=\frac{1}{\hat{\alpha}} \hat{V}^{-1}\left(\hat{\mu}-\hat{\beta} \mathbf{1}_{n}\right)$.

We can calculate the variance of these zero-risk premium portfolios. As the risk premia are reduced proportionally for all the risky assets in the worst 
scenario,

$$
\begin{aligned}
u^{* \prime} \hat{V} u^{*} & =\frac{\hat{\mu}^{\prime} \hat{V}^{-1} \hat{\mu}}{\hat{\mu}^{\prime} \hat{V}^{-1} \hat{\mu} \cdot \mathbf{1}_{n}^{\prime} \hat{V}^{-1} \mathbf{1}_{n}-\left(\mathbf{1}_{n}^{\prime} \hat{V}^{-1} \hat{\mu}\right)^{2}} \\
& =\frac{\mu^{\prime} \hat{V}^{-1} \mu}{\mu^{\prime} \hat{V}^{-1} \mu \cdot \mathbf{1}_{n}^{\prime} \hat{V}^{-1} \mathbf{1}_{n}-\left(\mathbf{1}_{n}^{\prime} \hat{V}^{-1} \mu\right)^{2}}
\end{aligned}
$$

The worst case covariance matrix is of form $\hat{V}^{-1}=V^{-1}+\hat{c} V^{-1} \mu \mu^{\prime} V^{-1}$, where $\hat{c}$ depends on $K$. Put it into the equation,

$$
\begin{aligned}
u^{* \prime} \hat{V} u^{*} & =\frac{\mu^{\prime} V^{-1} \mu\left(1+\hat{c} \mu^{\prime} V^{-1} \mu\right)}{\left(1+\hat{c} \mu^{\prime} V^{-1} \mu\right)\left(\mu^{\prime} V^{-1} \mu \cdot \mathbf{1}_{n}^{\prime} V^{-1} \mathbf{1}_{n}-\left(\mathbf{1}_{n}^{\prime} V^{-1} \mu\right)^{2}\right)} \\
& =\frac{\mu^{\prime} V^{-1} \mu}{\mu^{\prime} V^{-1} \mu \cdot \mathbf{1}_{n}^{\prime} V^{-1} \mathbf{1}_{n}-\left(\mathbf{1}_{n}^{\prime} V^{-1} \mu\right)^{2}},
\end{aligned}
$$

which is independent of $\hat{c}$ and hence independent of $K$.

To obtain a more robust and practical result for a financial economic model, we need to consider the effect of uncertainty on the model parameters. Tütüncü and Koenig (2004) introduces a simple way to model the uncertainty of input parameters in an asset allocation problem. They consider a range of parameters for both expected returns and covariances in a mean-variance model. The idea is logical and inspiring. However, there are still rooms for enhancement. In particular, this model cannot ensure that the covariance matrices in the range are invertible. It is not easy to find the optimal investment strategy unless there is no short-selling. A numerical method, interior point algorithm, is suggested to solve the problem in the general case. It is known that there might be infinitely many solutions. This is the difficulty of using a range of parameters in considering an asset allocation problem. One important advantage of our uncertainty model is that it takes the determinant of the covariance matrix into consideration. Therefore, it allows short selling but still has a unique explicit solution. Short selling is a useful strategy for risk hedging. For example, pair trading is popular among hedging funds and many professional investors. We know that many assets in the market are highly correlated and they can be used to hedge the unwanted risk of each other. The uncertainty approach can help those investors explore pair trading opportunities at the same time take care of the effect of uncertainty in the market. The model is also mathemati- 
cally tractable. Therefore, we can understand the financial meaning of this uncertainty model more easily and intuitively.

The model considers risk premium and covariance together with a single parameter. Thus, we can study their joint effect and differences. Our goal is to minimize the variance of the portfolio in the worst scenario for a target expected return. It is a saddle point problem and there exists a unique solution. The worst scenario is defined to be the measure in which the optimal portfolio has the greatest variance. Our analysis shows that the two sources of uncertainty have different mechanisms to increase the variance of the portfolio's return. They are different in nature. Covariance uncertainty increases the covariances among the assets, while, expected return uncertainty increases the leverage of the portfolio.

We use the worst scenario to illustrate the effect of uncertainty. There is another way to understand the meaning of worst scenario using Sharpe ratio. Sharpe ratio is the ratio of risk premium to standard deviation of the portfolio return. It measures the benefit of bearing investment risk and is known to be the price of risk. We continue to use $\mathcal{U}$ to denote the feasible set of parameters. In previous sections, it is obtained by a relative entropy constraints. Here, we consider a more general case and do not use the concept of relative entropy. We assume that $\mathcal{U}$ is convex, and independent of the investment strategy of the investors. We apply the mean-variance approach under the worst scenario to study the asset allocation problem in this new set of parameters. We apply the Lagrangian method on the expected return constraint. The problem becomes

$$
\min _{(\hat{\mu}, \hat{V}) \in \mathcal{U}}\left(\max _{u \in \mathbf{R}^{n}} u^{\prime} \hat{\mu}-\lambda u^{\prime} \hat{V} u\right) \quad \text { such that } u^{\prime} \hat{\mu} \geq r_{p} .
$$

Given the parameter $(\hat{\mu}, \hat{V})$, the optimal investment strategy is of this form,

$$
\hat{u}=\frac{1}{2 \lambda} \hat{V}^{-1} \hat{\mu}
$$

where $\lambda$ is the Lagrangian multiplier. With the expected return constraint under the worst scenario, we have

$$
\lambda=\frac{u^{* \prime} V^{*-1} u^{*}}{2 r_{p}} .
$$


Using the above results, the problem can be simplified

$$
\min _{(\hat{\mu}, \hat{V}) \in \mathcal{U}} \frac{r_{p} \hat{\mu}^{\prime} \hat{V}^{-1} \hat{\mu}}{2 u^{* \prime} V^{*-1} u^{*}} .
$$

Hence, the worst scenario minimizes $\hat{\mu}^{\prime} \hat{V}^{-1} \hat{\mu}$. On the other hand, due to the presence of the risk-free asset, the efficient frontier is a straight line. With the optimal investment strategy $\hat{u}$, we can find the Sharpe ratio of the efficient portfolios,

$$
\frac{\hat{u}^{\prime} \hat{\mu}}{\sqrt{\hat{u}^{\prime} \hat{V} \hat{u}}}=\sqrt{\hat{\mu}^{\prime} \hat{V}^{-1} \hat{\mu}} .
$$

Therefore, the worst scenario also minimizes the slope of the efficient frontier (the Sharpe ratio of these efficient assets). The result provides another financial interpretation of the worst scenario. It is the probability measure with the minimum reward on risk.

From the empirical analysis, we find that the effect of the expected return uncertainty is more substantial compared with the covariance uncertainty under the mean-variance framework. For a fixed required expected return under the worst scenario, the leverage on risky assets has a significant effect on the variance of the portfolio return. Modelling expected return uncertainty (e.g. Yang and Siu (2001), Maenhout (2004)) is a popular research topic because of its simplicity and relation with the Girsanov theorem. The numerical results rationalize the existing researches which focus on expected return uncertainty.

\section{Conclusions}

We have used the concept of relative entropy to model parameter uncertainty and studied the asset allocation problem. Two sources of uncertainty, risk premium uncertainty and covariance uncertainty, are studied in the model. Their effects and interaction are identified by considering the worst scenario. The explicit forms of the worst scenario and the optimal investment strategy are obtained. We have shown that relative entropy can ensure the invertibility of the covariance matrices in the set of parameters. It is vital for modelling covariance uncertainty because it can ensure the existence of a unique solution. The solution can also be found in a simpler way without using complicated numerical methods. In the world of uncertainty, 
people makes their investment decision with modified market parameters. Risk premia are reduced proportionally. Covariances are adjusted according to the risk premia. The influence of risk premium uncertainty is found to be stronger than covariance uncertainty due to the leverage effect. The behaviour of minimum variance frontiers of different uncertainty levels and the relationship between the worst scenario and Sharpe ratio are also discussed.

Acknowledgments. The authors are grateful for the detailed comments from the editor and two referees which help improving the manuscript substantially. Phillip Yam acknowledges the financial support from The Hong Kong RGC GRF 404012 with the project title: Advanced Topics In Multivariate Risk Management In Finance And Insurance, The Chinese University of Hong Kong Direct Grants 2010/2011 Project ID: 2060422 and 2011/2012 Project ID: 2060444. Phillip Yam also expresses his sincere gratitude to the hospitality of Hausdorff Center for Mathematics of the University of Bonn during the preparation of the present work. Hailiang Yang would like to thank the support from Research Grants Council of the Hong Kong Special Administrative Region (project No. HKU 705313P). Fei Lung Yuen would like to thank the financial support from Educational Institution Grant of the Society of Actuaries. Fei Lung Yuen would also like to express his sincere gratitude to the hospitality of the Department of Statistics and Actuarial Science of the University of Hong Kong and the Department of Statistics of the Chinese University of Hong Kong for the preparation of the present work. The research idea was initiated when the last author was at Heriot Watt University. 


\section{References}

Bertsimas, D., Brown, D. B. \& Caramanis, C. (2007). Theory and Application of Robust Optimization. SIAM Review, 53(3), 464-501.

Bertsimas, D., Lauprete, G. J. \& Samarov, A. (2004). Shortfall as a Risk Measure: Properties, Optimization and Applications. Journal of Economic Dynamics \& Control, 28, 1353-1381.

Bodnar, T., Parolya, N. \& Schmid, W. (2013). On the Equivalence of Quadratic Optimization Problems Commonly Used in Portfolio Theory. European Journal of Operational Research, 229, 637-644.

Boyd, S. \& Vandenberghe, L. (2004). Convex Optimization. (1st ed.). Cambridge: Cambridge University Press, (Chapters 4 and 5).

Calafiore, G. C. (2007). Ambiguous Risk Measures and Optimal Robust Portfolio. Society for Industrial and Applied Mathematics, 18(3), 853-877.

DeMigual, V. \& Nogales, F. J. (2009). Portfolio Selection with Robust Estimation. Operations Research, 57(3), 560-577.

Epstein, L. G. \& Schneider, M. (2008). Ambiguity, Information Quality, and Asset Pricing. The Journal of Finance, 63(1), 197-228.

Gennotte, G. (1986). Optimal Portfolio Choice Under Incomplete Information. The Journal of Finance, 41(3), 733-746.

Gregory, C., Darby-Dowman, K. \& Mitra, G. (2011). Robust Optimization and Portfolio Selection: The Cost of Robustness. European Journal of Operational Research, 212, 417-428.

Hansen, L. P. \& Sargent, T. J. (2001). Robust Control and Model Uncertainty. The American Economic Review, 91(2), 60-66.

Huang, X. \& Ying, H. (2013). Risk Index Based Models for Portfolio Adjusting Problem with Returns subject to Experts' Evaluations. Economic Modelling, 30, 61-66.

Klibanoff, P., Marinacci, M. \& Mukerji, S. (2005). A Smooth Model of Decision Making under Ambiguity. Econometrica, 73(6), 1849-1892. 
Lawson, J. \& Lim, Y. (2001). The Geometric Mean, Matrices, Metrics and More. The American Mathematical Monthly, 108(9), 797-812.

Li, D., Chan, T. F. \& Wan, W. L. (1998). Safety-First Dynamic Portfolio Selection. Dynamics of Continuous, Discrete and Impulsive Systems, 4, 585600 .

Li, D. \& Ng, W. L. (2000). Optimal Dynamic Portfolio Selection: Multiperiod Mean-Variance Formation. Mathematical Finance, 10(3), 387-406.

Lim, A. E. B. \& Shanthikumar, J. G. (2007). Relative Entropy, Exponential Utility, and Robust Dynamic Pricing. Operations Research, 55(2), 198-214.

Maenhout, P. J. (2004). Robust Portfolio Rules and Asset Pricing. The Review of Financial Studies, 17(4), 951-983.

Markowitz, H. (1952). Portfolio Selection. Journal of Finance, 7(1), 77-91.

Merton, R. C. (1972). An Analytical Derivation of the Efficient Portfolio Frontier, Journal of Financial and Quantitative Analysis, 7(4), 1851-1872.

Quiggin, J. (1982). A Theory of Anticipated Utility. Journal of Economic Behavior and Organization, 3, 323-343.

Schmeidler, D. (1989). Subjective Probability and Expected Utility without Additivity. Econometrica, 57(3), 571-587.

Scutellà, M. G., \& Recchia, R. (2013). Robust Portfolio Asset Allocation and Risk Measures. Annals of Operational Research, 204, 145-169.

Sharpe, W. F. (1964). Capital Asset Prices: A Theory of Market Equilibrium under Conditions of Risk. The Journal of Finance, 19(3), 425-442.

Tütüncü, R. H. \& Koenig, M. (2004). Robust Asset Allocation. Annals of Operations Research, 132, 157-187.

Tversky, A. \& Kahneman, D. (1992). Advances in Prospect Theory: Cumulative Representation of Uncertainty. Journal of Risk and Uncertainty, 5, 297-323.

Yaari, M. (1987). The Dual Theory of Choice under Risk. Econometrica, $55(1), 95-115$. 
Yang, H. \& Siu, T. K. (2001). Coherent Risk Measures for Derivatives under Black-Scholes Economy. International Journal of Theoretical and Applied Finance, 4(5), 819-835.

Yuen. F. L. \& Yang, H. (2012). Optimal Asset Allocation: A Worst Scenario Expectation Approach. Journal of Optimization Theory and Application, 153(3), 794-811.

Zhang, F. (2011). Matrix Theory: Basic Results and Techniques. (2nd ed.). New York: Springer, (Chapter 7). 